\title{
Prevalence and Life Style Determinants of Hypertension among Women in Iraq
}

\author{
Fadheel QJ* and Hussein AA \\ University of Kufa, Faculty of Pharmacy, Department of Pharmacology and \\ Toxicology, Iraq
}

*Corresponding author: Qayssar Joudah Fadheel, University of Kufa, Faculty of Pharmacy, Department of Pharmacology and Toxicology, Iraq, Email: qayssarj.fadheel@uokufa.edu.iq

\section{Abstract}

Back ground: Hypertension is one of the major prevalence chronic diseases in the world and it's greatly increase particularly in developing countries (Include Iraq) especially in women. A number of risk factors for increased the blood pressure include obesity resistance of insulin, excessive alcohol intake, elderly, sedentary life style, stress decrease intake of potassium and calcium, many of this factors are additives such as obesity and alcohol intake.

Aim of the study: The study was conducted with the aimed of determining the prevalence of Hypertension among woman and identifying life style related risk factor and we choose women because this town become endemic area with hypertension based on previous survey.

Patient: This is a randomize prospected, clinical study it was conducted in AL-Diwaniya teaching hospital, medicinal department, and emergency department. This study started at December 2015, this approved medical ethical community at AL-Kufa University.

Materials: There is considerable guidance about the range of appropriate devices for measuring blood pressure and about their maintenance and periodic recalibration local medical physics and biomedical/clinical engineering departments can often give further advice.

Methods: Sample of 60 case women in Al-Diwaniya city was chosen randomly all the women present in the hospital of AlDiwaniya Teaching Hospital at the time of visiting. Where interview according to specialise questionnaire... height.... weight; Blood pressure were measured.

Results: The prevalence lifestyle risk factor among the study population was as follow: Physical inactivity (65.4\%) (Over weight 39.4\%), (obesity 35.3), (OCP 16.4), (salty diet 16\%), (fatty diet 13.2\%) drug intake \{mostly (NASIDs) 11.4\}, (coffee intake 7.4\%), (smoking 1.5\%). A significant associated was found between some lifestyles risk factor and hypertension, these were drug intake and BMI, while no sign associated was found between other risk factor and hypertension which were, fatty diet, physical activity, coffee intake and OCP intake. 


\section{Open Access Journal of Pharmaceutical Research}

Conclusion: The prevalence of Hypertension was within the range of that reported for women in Iraq. A significant association was found between certain life style risk factor. Early detection for hypertension and education health program regarding life style behavior was high recommended.

Keywords: Hypertension; Obesity; Smoking; Alcohol; Physical Activity

\section{Introduction}

Hypertension is one of the important preventable causes of premature mortality and morbidity in the UK. Hypertension is a main risk factor for hemorrhagic and ischemic stroke, heart failure, myocardial infarction, cognitive decline, chronic kidney disease and premature death. Hypertension left without treatment is usually associated with a progressive rise in blood pressure. The vascular and renal damage that may cause culminate in a treatment-resistant state [1]. In many people with established primary hypertension, increased total peripheral resistance accounts for the increase in pressure while cardiac output remains within normal. There is fact that some younger people with 'borderline hypertension or prehypertension' have increase cardiac output, an increase heart rate and normal peripheral resistance, called hyperkinetic borderline hypertension [2] (Table 1).

\begin{tabular}{|c|c|c|}
\hline Category & Systolic, $\mathbf{m m H g}$ & Diastolic, $\mathbf{~ m m H g}$ \\
\hline Normal & $90-119$ & $60-79$ \\
\hline Prehypertension & $120-139$ & $80-89$ \\
\hline Stage 1 hypertension & $140-159$ & $90-99$ \\
\hline Stage 2 hypertension & $160-179$ & $100-109$ \\
\hline Stage 3 hypertension & $\geq 180$ & $\geq 110$ \\
\hline Isolated systolic hypertension & $\geq 140$ & $<90$ \\
\hline
\end{tabular}

Table 1: classification of hypertension for adult [3].

Hypertension results from a complex interaction of environmental factors and genes. Numerous common genetic variants with small influences on blood pressure have been identified as well as some rare genetic variants with large effects on blood pressure, but the genetic basis of hypertension is still poorly understood [4]. Secondary hypertension results from an identifiable cause. Kidney disease is the most common secondary cause of hypertension. Hypertension can also be caused by Endocrine conditions such as, hyperthyroidism, hypothyroidism, acromegaly, Conn's syndrome or hyperaldosteronism, hyperparathyroidism and pheochromocytoma.

Other causes of secondary hypertension include obesity, sleep apnea, pregnancy, coarctation of the aorta, excessive liquorice consumption and certain prescription medicines, herbal remedies and illegal drugs. Arsenic exposure through drinking water has been shown to correlate with elevated blood pressure [5].
Risk Factors of Hypertension (Being overweight or obese, Too much salt (sodium) in your diet, too little potassium in your diet, not being physically active 'drinking too much alcohol, stress' Non-steroidal Antiinflammatory Drugs (NSAIDs), Cough and Cold Medications, certain chronic conditions, a diet low in vitamin D [6-14].

\section{Management of Hypertension Includes}

Lifestyle changes to treat high blood pressure no matter what medications your doctor prescribes to treat your high blood pressure, you'll need to make lifestyle changes to lower your blood pressure. Your doctor may recommend several lifestyle changes, including: eating a healthier diet with less salt (the Dietary Approaches to Stop Hypertension, or DASH, diet) [15].

$\begin{array}{ll}- & \text { Exercising regularly } \\ - & \text { Quitting smoking } \\ \text { - } & \text { Limiting the amount of alcohol you drink } \\ \text { you're overweight or obese }\end{array}$




\section{Open Access Journal of Pharmaceutical Research}

\section{Pharmacological Treatment}

Antihypertensive therapy seeks to prevent the complications of high blood pressure, such as stroke and myocardial infarction. Evidence suggests that reduction of the blood pressure by $5 \mathrm{mmHg}$ can decrease the risk of stroke by $34 \%$, of ischaemic heart disease by $21 \%$, and reduce the likelihood of dementia, heart failure, and mortality from cardiovascular disease [16]. There are many classes of antihypertensive, which lower blood pressure by different means. Among the most important and most widely used drugs are thiazide diuretics, calcium channel blockers, ACE inhibitors, angiotensin II receptor antagonists (ARBs), and beta blockers [17].

\section{Materials and Methods}

This is a randomize prospected, clinical study it was conducted in Iraqi teaching hospitals, medicinal department, and emergency department. This study started at December 2015, this approved medical ethical community at University of Kufa.

\section{Materials}

There is considerable guidance about the range of appropriate devices for measuring blood pressure [18]. And about their maintenance and periodic recalibration Local medical physics and biomedical/clinical engineering departments can often give further advice [19].

Mercury sphygmomanometer: The mercury sphygmomanometer has been used for the traditional measurement of blood pressure. It is reliable and provides the reference standard for indirect measurement. However it is bulky, fragile and there are particular safety and economic concerns about the toxic effects of mercury. Mercury is being phased out of clinical use and mercury sphygmomanometers have already been removed from clinical areas in hospitals and primary care. Thus, alternatives to mercury sphygmomanometer are now required for routine clinical use [20]. Non-mercury devices that operate in a similar way to the traditional mercury column devices are available and provide a suitable alternative to mercury devices when manual auscultation is required to measure blood pressure.

Aneroid sphygmomanometers: Aneroid sphygmomanometers measure pressure using a lever and bellows system. They may be less accurate than mercury sphygmomanometers and their alternatives, especially over time. Using the manual auscultation technique they are subject to the same sources of observer error [21].

Automated devices: Automated devices are increasingly being used in hospitals and primary care. All sphygmomanometers need regular maintenance. Rubber tubing can crack and leak making cuff deflation hard to control, underestimating systolic and overestimating diastolic readings. Faulty valves can cause similar problems [21].

\section{Methods}

Sample of 60 case women in Al-Diwaniya city was chosen randomly all the women present in the hospital of Al-Diwaniya Teaching Hospital at the time of visiting. Where interview according to specialise questionnaire... height.... weight; Blood pressure were measured. The whole sample size was 60 participants; the only excluded character was pregnancy.

\section{Statistical Analysis}

Statistical analyses were performed using SPSS 16.0. An expert statistical advice was consulted for tests used. Data of quantitative variable were expressed as mean \pm SEM. Differences in each variable through treatment intervals in the same group were compared using paired sample students test. In all tests, $\mathrm{P}<0.05$ was considering to be statistically significant unless another level were stated.

\section{Results}

\section{Demographic disturb in health and patients with Hypertension}

In this table 2 below there were no significant between demographic patients with hypertension.

\begin{tabular}{|c|c|c|c|}
\hline Demographic Data & Health (mean \pm SEM) & H.T & P. Value \\
\hline Age & $60.22 \pm 1.86$ & $59.18 \pm 1.7$ & N.S \\
\hline Weight & $80.5 \pm 2.14$ & $81 \pm 2.16$ & N.S \\
\hline Height & $155.04 \pm 1.33$ & $154 \pm 1.22$ & N.S \\
\hline
\end{tabular}

Table 2: The mean of the Age, Weight, and Height among healthy and patient with Hypertension.

Patient with Hypertension expressed in mean \pm SEM; N.S: N0 Significant; SEM: standard error mean. 


\section{Open Access Journal of Pharmaceutical Research}

\section{Relationship between different Risk Factors among Healthy and Patient with Hypertension (Table 3)}

\begin{tabular}{|c|c|c|c|}
\hline \multirow{2}{*}{ Risk Factors } & \multicolumn{2}{|c|}{ Hypertension } & \multirow{2}{*}{ P. value } \\
\hline & Number & $\%$ & \\
\hline \multicolumn{3}{|c|}{ 1. Physical inactivity } & \multirow{4}{*}{$<0.001$} \\
\hline Inactive & 39 & 65.4 & \\
\hline Moderate & 11 & 18.3 & \\
\hline Active & 10 & 16.3 & \\
\hline \multicolumn{3}{|l|}{ 2. B.M.I } & \multirow{4}{*}{$<0.001$} \\
\hline Obesity & 21 & 35.3 & \\
\hline Over weight & 23 & 39.6 & \\
\hline Normal & 16 & 25.1 & \\
\hline \multicolumn{3}{|c|}{ 3. Contraceptive } & \multirow{3}{*}{ N.S } \\
\hline Positive & 10 & 16.4 & \\
\hline Negative & 50 & 83.6 & \\
\hline \multicolumn{3}{|c|}{ 4. Salt diet intake } & \multirow{3}{*}{$<0.05$} \\
\hline evtiisoP & 9 & 16 & \\
\hline Negative & 51 & 84 & \\
\hline \multicolumn{3}{|c|}{ 5. Fatty diet intake } & \multirow{3}{*}{ N.S } \\
\hline evtiisoP & 8 & 13.2 & \\
\hline Negative & 52 & 86.8 & \\
\hline \multicolumn{3}{|l|}{ 6. Drug uses } & \multirow{3}{*}{$<0.001$} \\
\hline Positive & 7 & 11.4 & \\
\hline Negative & 53 & 88.6 & \\
\hline \multicolumn{3}{|c|}{ 7. Coffee intake } & \multirow{3}{*}{ N.S } \\
\hline Positive & 4 & 7.4 & \\
\hline Negative & 56 & 92.6 & \\
\hline \multicolumn{3}{|l|}{ 8. Smoker } & \multirow{3}{*}{ N.S } \\
\hline Positive & 1 & 1.5 & \\
\hline Negative & 59 & 98.5 & \\
\hline
\end{tabular}

Table 3: Relationship between lifestyle result factors and Hypertension.

\section{The Prevalence Lifestyle Risk Factor among the Study Population}

Physical inactivity (65.4\%) (Over weight 39.4\%), (obesity 35.3), (OCP 16.4), (salty diet 16\%), (fatty diet $13.2 \%$ ) drug intake \{mostly (NASIDs) 11.4 \}, (coffee intake 7.4\%), (smoking 1.5\%). A significant associated was found between some lifestyles risk factor and hypertension, these were drug intake and BMI, while no sign associated was found between other risk factor and hypertension which were, fatty diet, physical activity, coffee intake and oral contraceptive pill intake.

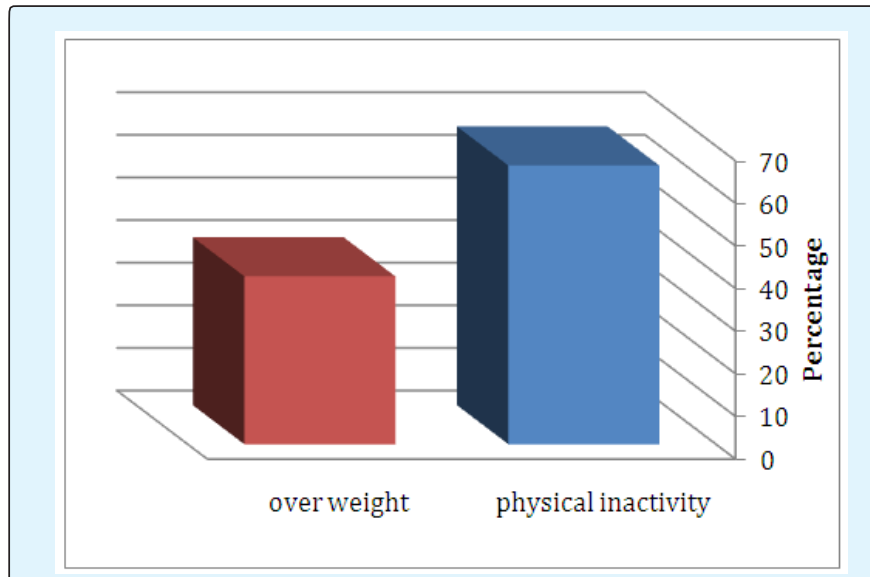

Figure 1: A comparison of prevalence between Physical inactivity and overweight among study population.

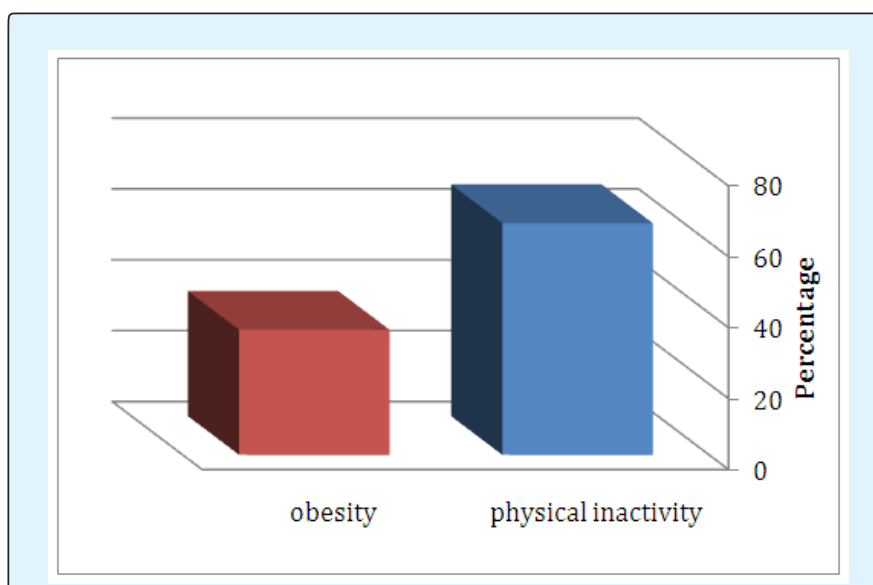

Figure 2: A comparison of prevalence between Physical inactivity and Obesity among study population.

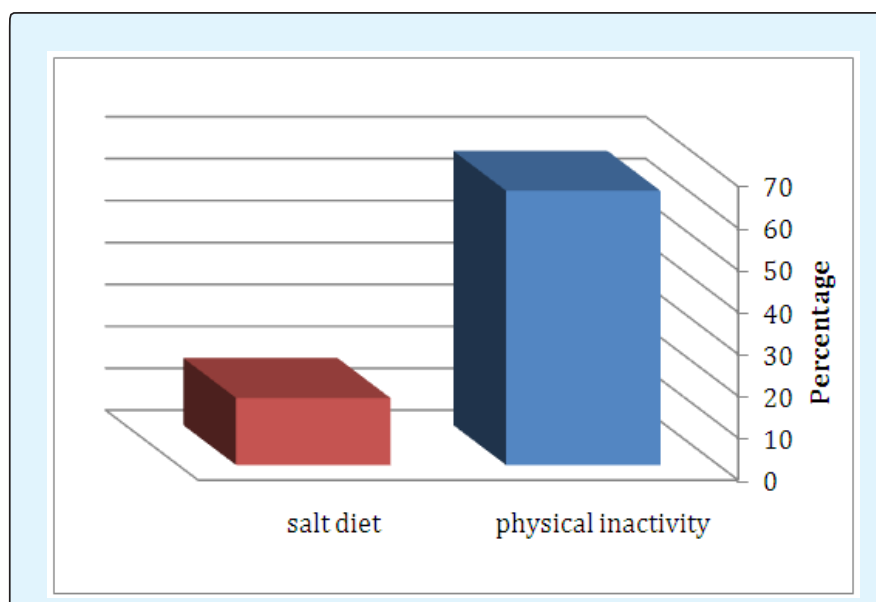

Figure 3: A comparison of prevalence between Physical inactivity and salt diet among study population. 


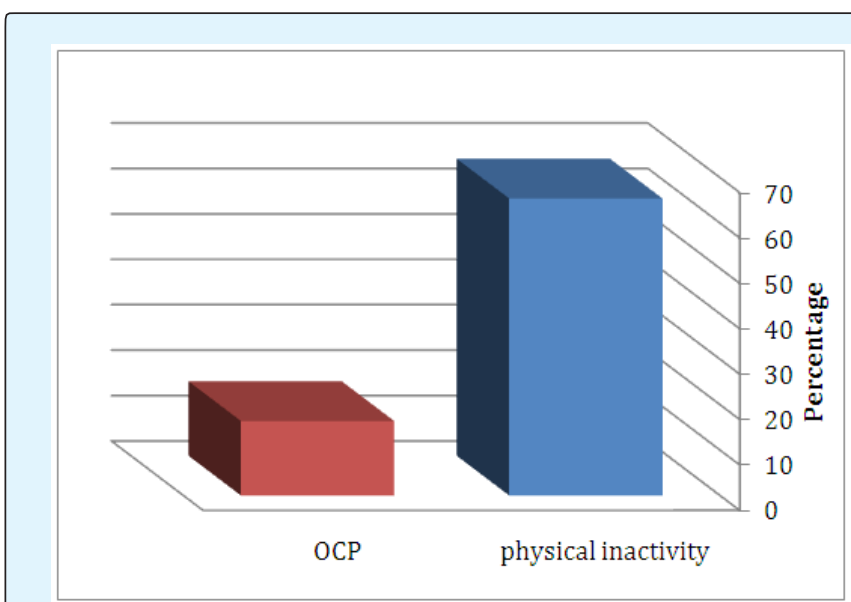

Figure 4: A comparison of prevalence between physical inactivity and OCP among study population.

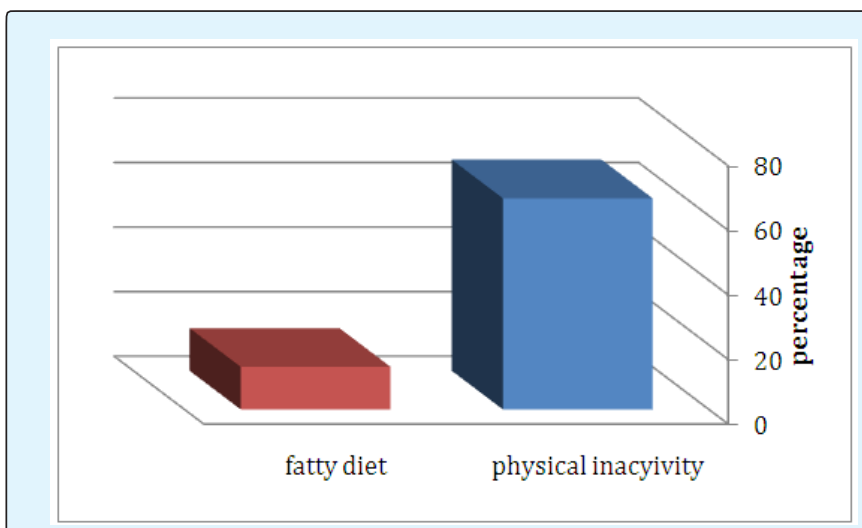

Figure 5: A comparison of prevalence between Physical inactivity and Fatty Diet among study population.

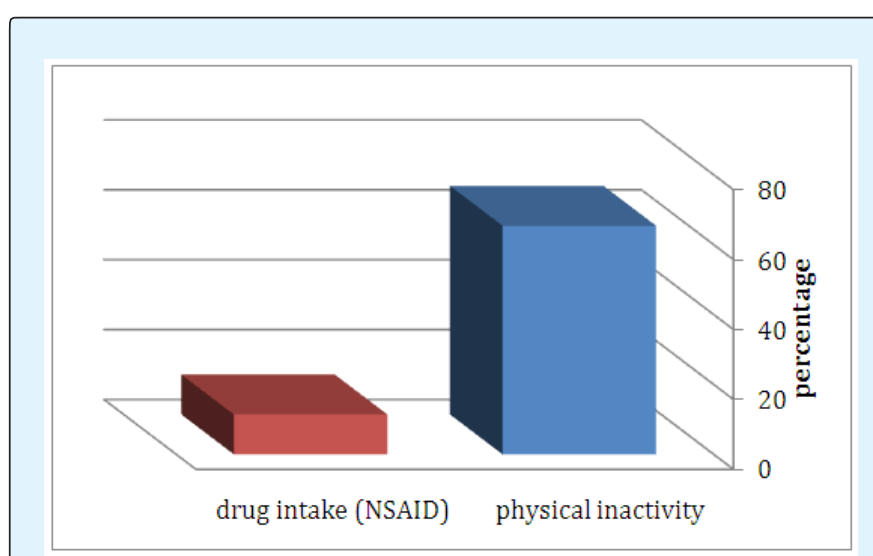

Figure 6: A comparison of prevalence between Physical inactivity and Drug intake among study population.

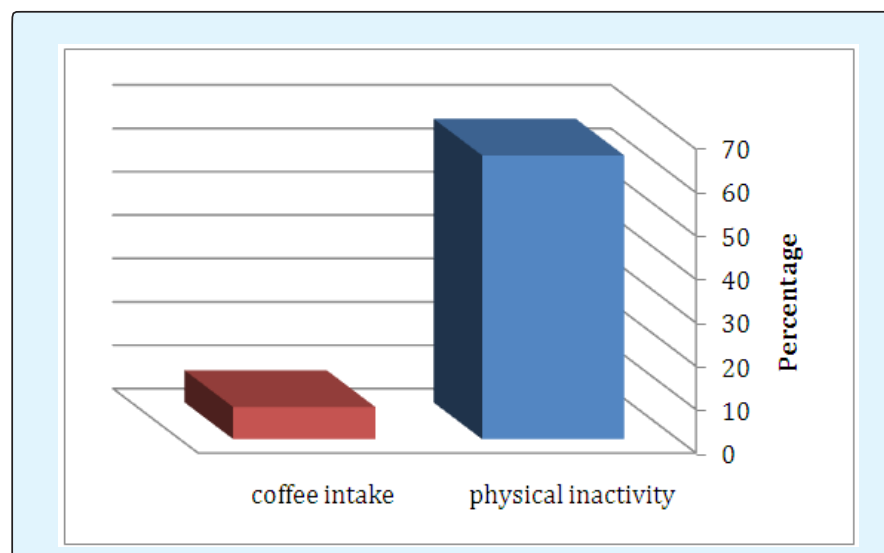

Figure 7: A comparison of prevalence between Physical inactivity and coffee intake among study population.

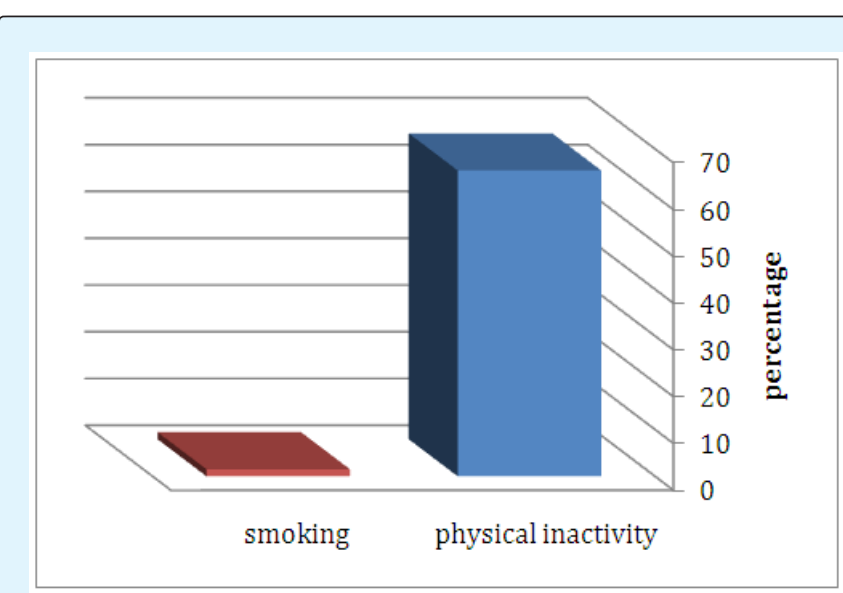

Figure 8: A comparison of prevalence between physical inactivity and Smoking among study population.

\section{Discussion}

Hypertension is a major risk factor for stroke (ischaemic and hemorrhagic), myocardial infarction, heart failure, chronic kidney disease, peripheral vascular disease, cognitive decline and premature death. Untreated hypertension is associated a progressive rise in blood pressure, often culminating in a treatment resistant state due to associated vascular and renal damage. Blood pressure is quantified as diastolic and systolic pressures measured in millimeters of mercury (mmHg). The diastolic pressure represents the pressure during ventricular relaxation in diastole whereas the systolic pressure represents the peak pressure due to ventricular contraction during systole. Either or both pressures have specified upper limits of normal and elevation in either or both pressures are used to define hypertension [22]. 


\section{Open Access Journal of Pharmaceutical Research}

\section{Effect of Physical Inactivity on Hypertension}

The present study show a significant effect of this risk factor ( $p$-value $<0.05$ ), this findings are in agreement with results of study, which stated that people who are inactive tend to have higher heart rates [23]. The higher your heart rate, the harder your heart must work with each contraction and the stronger the force on your arteries. Lack of physical activity also increases the risk of being overweight.

\section{Effect of Smoking on Hypertension}

The result of current study show is no significant effect of smoking on hypertension, this finding are in consistency with results of study, which stated that smoking temporarily raises blood pressure and increases your risk of damaged arteries [24]. The use of tobacco can be devastating to your health, especially if you're already at risk for high blood pressure. Secondhand smoke exposure to other people's smoke increases the risk of heart disease for nonsmokers.

\section{Effect of Overweight and Obesity on Hypertension}

The present study show a significant effect of these risk factors on hypertension ( $p$-value $<0.05$ ), this finding are in similarity with study which stated that being overweight increases your chances of developing high blood pressure [24]. A body mass index between 25 and 30 is considered overweight. A body mass index over 30 is considered obese. About two-thirds of U.S. adults are overweight or obese. About one in three U.S. children ages 2 to 19 are overweight or obese. Excess weight increases the strain on the heart, raises blood cholesterol and triglyceride levels, and lowers HDL (good) cholesterol levels. It can also make diabetes more likely to develop. Losing as little as 10 to 20 pounds can help lower your blood pressure and your heart disease risk. To successfully and healthfully lose weight and keep it off most people need to subtract about 500 calories per day from their diet to lose about one pound per week.

\section{Effect of Contraceptives on Hypertension}

This study show no significant effect of this risk factor, this finding are in consistency with study which stated that oral contraceptive and postmenopausal therapy may be induce HTN by many mechanisms such as rennin angiotensin system increase substrate that lead to increase in amount of angiotensin and cause decrease in suppression of rennin release, sodium \& water retention [25]. Numerous studies show that chronic administration of contraceptive containing estrogen \& progesterone may increase blood pressure \&the risk of cardiovascular.

\section{Effect of Salt Diet Intake on Hypertension}

The current study is a significant effect of this risk factor $(<0.05)$, this finding are is similarity with study which stated that a high salt diet intake disrupts the natural balance in the body [26]. This causes fluid retention which increases the pressure against blood vessels. For every one gram cut from our average daily intake, there would approximately 6000 fewer deaths from strokes and heart attacks every year.

\section{Effect of Fatty Diet Intake on Hypertension}

The current study is no significant effect of this risk factor. This finding is in agreement with studies which stated that consumption of food that are high in fat result in increased absorption of cholesterol [27]. Fatty foods also contain high amount of triglyceride. Fat molecule is similar to cholesterol and saturated fats. Fat food restaurants are major supplier of fatty foods in United States. Fat consumption yields twice the amount of calories in comparison to protein or carbohydrate intake. Fatty foods affect blood pressure by influencing the rate of atherosclerosis (increase thickness of arterial walls that result from the accumulation of cholesterol within the arterial wall).

\section{Effect of Drug Uses on Hypertension}

The current study show a significant effect of this risk factor on hypertension ( $p$-value $<0.05$ ), this finding are in similarity with finding of study which stated that a number of medication \& other condition can causes HTN and called secondary hypertension [27]. For example cough \&cold medication (including decongestant), analgesic \& birth controls pill can cause blood pressure to increase.

\section{Effect of Coffee Intake on Hypertension}

The present study show no significant effect of this risk factor on hypertension, this finding are in similarity with result of study which show that according to studies take on women, the risk of hypertension may be lower in coffee abstainers [25]. Studies show that coffee intake around 5 cups per day cause small elevation in BP (approximately 2/1 mmhg) when compared to abstinence or use of decaffeinated coffee. However there are many other substance in coffee such as polyphenols, soluble fiber and potassium, which could exert a beneficial effect in the cardiovascular system. Although the precise nature of the relation between coffee and BP is still unclear, most 


\section{Open Access Journal of Pharmaceutical Research}

evidence suggest that regular intake of caffeinated coffee does not increase the risk of hypertension.

\section{Conclusion}

From the results of this study we can conclude the following: the prevalence of Hypertension was within the range of that reported for women in Iraq. A significant association was found between certain life style risk factor. Early detection for Hypertension and education health program regarding life style behavior was high recommended.

\section{References}

1. Mendis Shanthi, Puska Pekka, Norrving, Bo (2011) Global atlas on cardiovascular disease prevention and control (PDF) 1st (edn.) Geneva: World Health Organization in collaboration with the World Heart Federation and the World Stroke Organization.

2. Struijker Boudier HA, le Noble JL, Messing MW, Huijberts MS, le Noble FA, et al. (1992) The microcirculation and hypertension. J Hypertens Suppl 10(7): S147-156.

3. Luma GB, Spiotta RT (2006) Hypertension in children and adolescents. Am Fam Physician 73(9): 15581568.

4. Lifton RP, Gharavi AG, Geller DS (2001) Molecular mechanisms of human hypertension. Cell 104(4): 545-556.

5. Grossman E, Messerli FH (2012) Drug-induced Hypertension: An Unappreciated Cause of Secondary Hypertension. Am J Med 125(1): 14-22.

6. Stokkermans TJ, Dunbar MT (1998) Solar retinopathy in a hospital-based primary care clinic. J Am Optom Assoc 69(10): 625-636.

7. Roalfe AK, Holder RL, Wilson S (2008) Standardisation of rates using logistic regression: a comparison with the direct method. BMC Health Serv Res 8: 275.

8. Basu S, Millett C (2013) Social epidemiology of hypertension in middle-income countries: determinants of prevalence, diagnosis, treatment, and control in the WHO SAGE study. Hypertension 62(1): 18-26.
9. (2009) Causes and Risk Factors. Diabetic Retinopathy. United States National Library of Medicine.

10. Kertes PJ, Johnson TM (2007) Evidence Based Eye Care. Philadelphia, PA: Lippincott Williams \& Wilkins.

11. Wong TY, McIntosh R (2005) Hypertensive retinopathy signs as risk indicators of cardiovascular morbidity and mortality. Br Med Bull (73-74): 57-70.

12. Barnaby AM, Hansen RM, Moskowitz A, Fulton A (2007) Development of scotopic thresholds in retinopathy of prematurity. Invest Ophthalmol Vis Sci 48(10): 4854-4860.

13. Chen JC, Lee LR (2004) Solar retinopathy and associated optical coherence tomography findings. Clin Exp Optom 87(6): 390-393.

14. WHO (2015) World Health Statistics.

15. James PA, Oparil S, Carter BL, Cushman WC, Dennison Himmelfarb C, et al. (2013) 2014 Evidence-Based Guideline for the Management of High Blood Pressure in Adults: Report From the Panel Members Appointed to the Eighth Joint National Committee (JNC 8). JAMA 311(5): 507-520.

16. (2013) Antihypertensive Agents at the US National Library of Medicine Medical Subject Headings.

17. Law M, Wald N, Morris J (2003) Lowering blood pressure to prevent myocardial infarction and stroke: a new preventive strategy. Health Technol Assess $7(31): 1-94$.

18. British Hypertension Society (2010) Validated devices suitable for home use.

19. (2000) Medical Devices and Equipment Management: Repair and Maintenance Provision (MDA DB2000 (02) London: Department of Health.

20. Brien OE (2001) State of the market for devices for blood pressure measurement. Blood Pressure Monitoring 6(6): 281-286.

21. Beevers G, Lip G, Brien E (2001) ABC of hypertension: Blood pressure measurement. Part II-conventional sphygmomanometry: technique of auscultatory blood pressure measurement. BMJ 322(7293): 1043-1047. 


\section{Open Access Journal of Pharmaceutical Research}

22. (1985) Cardiovascular risk and risk factors in a randomized trial of treatment based on the betablocker oxprenolol: the International Prospective Primary Prevention Study in Hypertension (IPPPSH) The IPPPSH Collaborative Group. J Hypertens 3(4): 379-392.

23. Peter JW (2003) Retinopathy. BMJ 326(7395): 924.

24. Poulter NR, Prabhakaran D, Caulfield M (2015) Hypertension. Lancet 386 (9995): 801-812.
25. Woods JW (1988) oral contraceptive and Hypertension. Hypertension 11(3-2): 11-15.

26. MacGregor GA (1999) Nutrition and blood pressure. Nutri metab cardiovas Dis 9(4S): 6-15.

27. Sukhinder Cheema (2006) Advances in biochemistry in health and disease. Biochemistry of atherosclerosis. Oxidized LDL and antioxidants in atherosclerosis. 